\title{
Effects of Resistance Exercise on Intramuscular Oxygenation and Muscle Fiber Composition
}

Fumio Aita ${ }^{1}$, Shukoh Haga², Shogo Sato ${ }^{3}$, Takuya Sakurai ${ }^{3^{*}}$, Kazuki Esaki ${ }^{4}$, Takafumi Hamaoka ${ }^{5}$, Masao Mizuno ${ }^{6}$, Koji Toshinai ${ }^{7}$, Hiromi Miyazaki $^{8}$, Tohru Takemasa ${ }^{9}$, Noboru Hashimoto ${ }^{10}$, Junetsu Ogasawara ${ }^{3}$, Toshihito Katsumura ${ }^{11}$, Takako Kizaki ${ }^{3}$ and Hideki Ohno $^{3}$

${ }^{1}$ Edosaki Sohgoh High School, Inashiki, Japan

${ }^{2}$ Institute of Health and Sport Science, University of Tsukuba, Tsukuba, Japan

${ }^{3}$ Department of Molecular Predictive Medicine and Sport Science, Kyorin University, School of Medicine, Mitaka, Japan

${ }^{4}$ Faculty of Education, Shiga University, Ohtsu, Japan

${ }^{5}$ Faculty of Sports and Health Science, Ritsumeikan University, Kusatsu, Japan

${ }^{6}$ Faculty of Education, Hokkaido University, Sapporo, Japan

${ }^{7}$ Faculty of Medicine, University of Miyazaki, Miyazaki, Japan

${ }^{8}$ Division of Traumatology, National Defense Medical College Research Institute, Tokorozawa, Japan

${ }^{9}$ Graduate School of Comprehensive Human Sciences, University of Tsukuba, Tsukuba, Japan

${ }^{10}$ Department of Judo Therapy, Faculty of Health Science, Tokyo Ariake University of Medical and Health Science, Kotoh-ku, Japan

${ }^{11}$ Department of Sports Medicine for Health Promotion, Tokyo Medical University, Shinjuku-ku, Japan

\begin{abstract}
Determining the oxygenation level of skeletal muscle is an effective non-invasive method of evaluating its physiological changes. Nevertheless, the relationship between muscle fiber composition and intramuscular oxygenation is still unclear. In the present study we examined the differences in muscle fiber composition and their relationship to muscle oxygenation levels by comparing power lifters (Training group), who regularly engaged in muscular resistance training, with ordinary healthy people (Control group). The vastus lateralis muscle oxygenation level was measured via near infrared continuous wave spectroscopy, and muscle tissues were collected and used for histochemical analyses in order to calculate muscle fiber compositions. When the rate of decrease in muscle oxygenation after performing a single squat at 50 and $80 \%$ of the participants' maximal lifting weight (MLW) was examined, the rate of decrease was significantly higher at $80 \%$ of MLW than at $50 \%$ of MLW in Training group, and was significantly higher in the Training group than in the Control group. Moreover, after performing multiple squat movements at $80 \%$ of MLW, compared with the Control group, the Training group showed a significant delay in the time it took for their muscle oxygenation level to recover to $50 \%$ of its original level at resting time $\left(\mathrm{T}_{1 / 2}\right)$. A significant correlation between the occupancy of Type Ila fiber and $T_{1 / 2}$, or between an average cross-sectional area of muscle fibers and $T_{1 / 2}$ was noted. The present study demonstrated that in the Training group, in a cross-sectional area of Type lla fiber that had increased, the decreased muscle oxygenation level due to performing squat exercises exhibited a delay in recovery at the resting time, suggesting that the amount of oxygen consumption was increased in the Training group because the crosssectional area of Type lla fiber was larger, compared with the Control group.
\end{abstract}

Keywords: Resistance training; Muscle oxygenation; Near infrared continuous wave spectroscopy; Muscle fiber composition

\section{Introduction}

Skeletal muscle is composed of several types of muscle fibers such as Type I, Type IIa, and Type IIx, all of which differ in contractile and metabolic characteristics. Skeletal muscle is referred to as a "plastic" tissue because it adapts to exercise. In fact, the exercise-induced stimulation from muscular resistance increases muscle mass and strength, changes muscle composition and improves muscle function. This process is thought to be the after-effect of hypertrophy of muscle fiber size, an increase in muscle fiber number, accretion of connective tissue around muscle fiber, or some combination of these [1].

Recently, the effects of hypoxia on muscle structure and function have been investigated. Resistance training under local hypoxia reportedly induces muscle hypertrophy (Type II fiber) and increases muscle strength [2-5]. Also, it has been suggested that under hypoxia, low-intensity resistance training, which is generally thought not to induce muscle hypertrophy, can induce muscle hypertrophy [6,7]. On the other hand, hypoxia-induced intramuscular environmental changes are synergistically developed by resistance exercise [8]. A muscle contraction-induced rise in intramuscular pressure excretes blood and results in the flow of more blood after relaxation. Thus, the ischemic interval is dependent upon the length of the muscle contractile time. Therefore, the extension of an ischemic interval induced by resistance training attracts hypoxia and affects muscle structure and function.

Examination of muscle oxygenation levels is thought to be effective as a non-invasive method of evaluating physiological changes in skeletal muscle caused by exercise. Near infrared continuous wave spectroscopy (NIRcws) has been used to evaluate the kinetics of skeletal muscle oxygenation during various types of exercise non-invasively and continuously [9-11]. In fact, some studies have examined the relationship between changes in exercise intensity and muscle oxygenation level by having participants perform squat exercises [12-15]. For example, muscle oxygen recovery kinetics appeared to be influenced by differences in the intensity and volume of exercise [12]. Nevertheless, in addition to exercise intensity and volume, little is known about factors that affect changes in muscle oxygenation. In the present study, we hypothesized that muscle fiber composition affected intramuscular oxygenation because of differences in oxygen consumption and oxidation capacity of each muscle fiber type. Therefore, we investigated differences in muscle

*Corresponding author: Takuya Sakurai, Department of Molecular Predictive Medicine and Sport Science, Kyorin University, School of Medicine, 6-20-2 Shinkawa, Mitaka, Tokyo 181-8611, Japan, Tel: +81(422)5511; Fax: +81(422)44 4427; E-mail: sakutaku@ks.kyorin-u.ac.jp

Received November 05, 2013; Accepted November 29, 2013; Published December 06, 2013

Citation: Aita F, Haga S, Sato S, Sakurai T, Esaki K, et al. (2013) Effects of Resistance Exercise on Intramuscular Oxygenation and Muscle Fiber Composition. J Sports Med Doping Stud 3: 133. doi:10.4172/2161-0673.1000133

Copyright: (c) 2013 Aita F, et al. This is an open-access article distributed under the terms of the Creative Commons Attribution License, which permits unrestricted use, distribution, and reproduction in any medium, provided the original author and source are credited. 
fiber compositions and their relationship with muscle oxygenation levels were evaluated via NIRcws between powerlifters (Training group) and ordinary healthy people (Control group).

\section{Materials and Methods}

\section{Participants}

Thirteen healthy male adults participated in the study: 7 powerlifters for the Training group and 6 healthy graduate students and working adult volunteers for the Control group. The powerlifters belonged either to a powerlifting club at the University of Tsukuba in Japan or to a powerlifting association in Japan, and had entered a powerlifting competition. The average length of competitive experience in powerlifting of the Training group was $8.0 \pm 2.8$ years. All the participants had almost the same age, weight, BMI, and skinfold thickness, except for height (Table 1). The study was conducted with the participants who agreed to participate after having received sufficient explanation on the purpose, method, safety, and problems of this study. Experimental procedures were approved by the Ethics Committee of the Institute of Health and Sport Science, University of Tsukuba, Japan.

\section{Protocol for measuring}

The protocol of this research is shown in Figure 1. After a muscle biopsy, the participants were given time to recover until muscular activities could be done smoothly. We determined the maximal lifting weight (MLW) of each participant in advance and calculated relative exercise loads at 50 and $80 \%$ of their MLW. The determination of the MLW for the powerlifters was based on their best records in a powerlifting competition. On the other hand, for determinations of MLW for the Control group, we measured each of 6 repetition maximum (RM) loads during a squat exercise. A 6RM load is known to be reliable (Intra-class correlation coefficient $>0.95$ ) and can be an alternative to a 1RM load determination [16]. The 1RM load can be estimated from a $6 \mathrm{RM}$ load using the following conversion equation: $1 \mathrm{RM}$ load=6RM load $/ 85 \%$ [17].

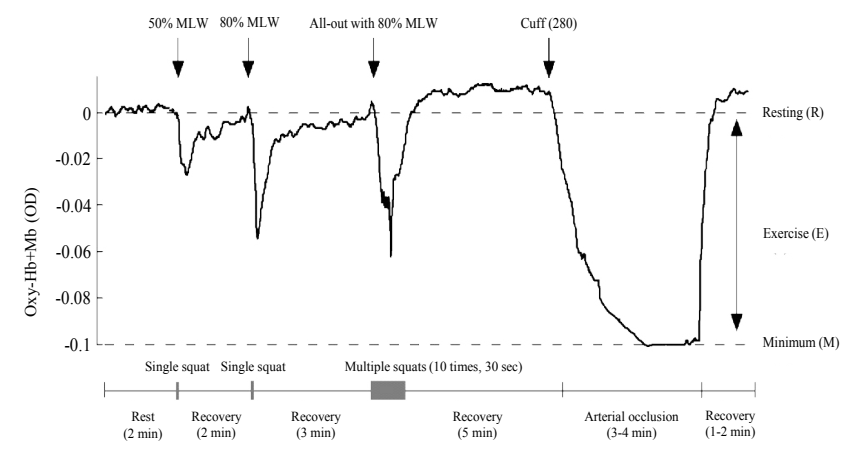

Figure 1: Experimental protocol of the muscle oxygenation during a squat exercise

Relative muscle oxygenation was determined by assigning the resting oxy$\mathrm{Hb}+\mathrm{Mb}$ value $(\mathrm{R})(100 \%)$ and the minimum oxy- $\mathrm{Hb}+\mathrm{Mb}$ value $(\mathrm{M})$ obtained from arterial occlusion ( $0 \%)$. Muscle oxygenation (\%) was expressed by using algebraic expression, i.e., muscle oxygenation $(\%)=(M-E) /(M-R) \times 100$. MLW $=$ maximal lifting weight.
The participants took a standing posture in the squat rack (NISHI Athletic Goods Co., Ltd., Tokyo, Japan) after resting on a chair for 2 minutes. Each participant assumed their exercise posture 10 seconds before the start of the exercise. At the moment when they were given a signal, they started a squat movement at $50 \%$ of their MLW. Next, after confirming that the muscle oxygenation level had recovered to the resting level (almost $2 \mathrm{~min}$ ), the participants performed a squat at $80 \%$ of their MLW. Finally, after sufficient recovery time (almost 3 min), the participants performed multiple squats at $80 \%$ of their MLW until exhaustion (almost 10 times, $30 \mathrm{sec}$ ). We judged subjects as being in an exhausted state when failure to perform the squat exercise (failure to stand, decreases in knee flexion angle, stagger, and so on) was observed. Because the Control groups lacked sufficient experience in squat exercises, assistants with sufficient knowledge and experience, supervised the exercise performances of the Control group to avoid accidents during exercise. Moreover, immediately after each squat movement, assistants on both sides of a participant lifted the barbell by hand so that the participant could be in the recovery period in a relaxed condition. This was done to avoid the influence of muscle oxygenation during the recovery process in participants after the exercises. Five minutes after the completion of all the exercises, arterial occlusion was performed.

\section{Evaluation of oxygenation levels in skeletal muscle}

NIRcws is used as the measurement of oxygen concentration in tissues by utilizing the absorbance characteristics of hemoglobin and myoglobin at the near infrared region. To measure NIRcws, we used Omron's near-infrared spectroscopy device (HEO-100, Kyoto, Japan). This device uses a two-wavelength method of two near infrared wavelengths (760 and $850 \mathrm{~nm}$ ) as the basis of measurement [18]. Its probe is composed of two LEDs, which emit two near infrared wavelengths, photo diode, and photodetection amplifier. The probe was attached to the vastus lateralis muscle of the right thigh, which was the same part where the muscle biopsy was conducted. Near-infrared light of 760 and $850 \mathrm{~nm}$ wavelengths emitted from the light source was injected into the muscle tissue and changes in oxygenation levels of skeletal muscle were measured based on the amount of light that reached the photodetector sensor after being absorbed by oxyhemoglobin and oxymyoglobin (oxy- $\mathrm{Hb}+\mathrm{Mb})$, as well as deoxyhemoglobin and deoxymyoglobin (deoxy- $\mathrm{Hb}+\mathrm{Mb}$ ). A relative value of skeletal muscle oxygenation cannot be calculated by the measurement of oxy- $\mathrm{Hb}+\mathrm{Mb}$ using NIRcws. Therefore, following the arterial occlusion method $\geq 280$ $\mathrm{mmHg}$ of pressure was applied to the thigh cuffs until the oxy-Hb+Mb showed the minimal value [19-21]. The minimal oxy- $\mathrm{Hb}+\mathrm{Mb}$ value was determined when the oxy- $\mathrm{Hb}+\mathrm{Mb}$ value had reduced (almost $2 \mathrm{~min}$ ) and maintained a steady state for 1-2 min. The relative value of skeletal muscle oxygenation was calculated with the following formula, using the minimum value obtained $(M)$, the value obtained at resting $(R)$, and the value obtained during exercise $(\mathrm{E})$ :

\section{(Formula) Muscle oxygenation $(\%)=(\mathrm{M}-\mathrm{E}) /(\mathrm{M}-\mathrm{R}) \times 100$.}

The distance between the light source of the probe and the photodetector used was $3 \mathrm{~cm}$. Therefore, the depth of measurement was assumed to be 1 to $2 \mathrm{~cm}$ below the skin surface [21-23]. Based on the fact that the thigh skinfold thickness in subjects tested in this

\begin{tabular}{|c|c|c|c|c|c|c|}
\hline & Age (yr) & Height (cm) & Weight (kg) & BMI $\left(\mathrm{kg} / \mathrm{m}^{2}\right)$ & Skinfold thickness $(\mathrm{cm})$ & Training experience (yr) \\
\hline Control group $(\mathrm{N}=6)$ & $33.2 \pm 5.1$ & $171.4 \pm 0.7$ & $71.4 \pm 1.9$ & $24.3 \pm 0.7$ & $0.5 \pm 0.1$ & - \\
\hline Training group $(\mathrm{N}=7)$ & $30.0 \pm 4.8$ & $165.5 \pm 2.2^{*}$ & $68.7 \pm 4.4$ & $25.1 \pm 1.4$ & $0.4 \pm 0.1$ & $8.0 \pm 2.8$ \\
\hline
\end{tabular}

Values are means \pm SE. BMI = body mass index; skinfold thickness $=$ thickness of skin and tissue at the place of NIRcws probe. ${ }^{*} p<0.05$ vs. Control group.

Table 1: Characteristics of subjects. 
experiment averaged $0.4 \pm 0.1 \mathrm{~cm}$ for the Training group and $0.5 \pm 0.1$ $\mathrm{cm}$ for the Control group, respectively, it could be assumed that the oxygen levels measured were derived mainly from skeletal muscle.

\section{Muscle biopsy}

A physician who specialized in exercise physiology conducted the muscle biopsies. Dexterity with vastus lateralis muscle was the focus of this experiment. Skin was sterilized with an anti-bacterial preparation (Isodine) and then muscle tissue $(20 \sim 30 \mathrm{mg})$ was sampled under local anesthesia (1\% xylocaine). Two specimens of muscle tissue were sampled for histochemical analysis. The muscle tissue was rapidly embedded in a compound (TISSUE-TEK, Miles Co., Elkhart, USA) and instantly frozen in 2-methylbutane (isopentane; WAKO, Osaka, Japan), then maintained with liquid nitrogen. Frozen samples were stored at $-80^{\circ} \mathrm{C}$ until moments before use.

\section{Histochemical analysis}

A continuous cross-section $(10 \mu \mathrm{m})$ of muscle tissues was obtained by cryostat (OF/FAS5030; Bright Instrument, Cambridgeshire, UK) at $-20^{\circ} \mathrm{C}$. The sections were subjected to myosin-ATPase stain (preincubation; $\mathrm{pH} 10.3$ and 4.6) [2,24]. The sections were photographed using a photomicrographic camera (Nikon M-35FA, Nikon, Tokyo, Japan). According to methods established by Brooke and Kaiser each sample of muscle fiber was separated into either Type I or Type II (Type IIa, Type IIx, and Type IIc) fiber, and the $\%$ of Fiber Type was calculated. Each type of muscle fiber from the same sample was randomly traced and calculated as to mean fiber area per fiber with respect to each of the types using image analysis software $[3,25]$. Based on those values, the muscle fiber cross-sectional area per capillary vessel (i.e., Diffusion index between capillary vessel and muscle fiber and muscle fiber type area distribution) was also calculated. On the other hand, other sections were subjected to amylase-PAS stain (pre-incubation; 0.5\% a-amylase) for visualization of capillaries [26]. The number of capillaries around the fiber type and the number of capillaries per unit area $\left(1000 \mu \mathrm{m}^{2}\right)$ for each fiber type were calculated.

\section{Statistical analysis}

The numerical values obtained are indicated as means \pm SE. An unpaired t-test was applied to compare the data between the two groups. Furthermore, Pearson's correlation analysis was conducted to examine the correlation between different measured values. The level of statistical significance was set at less than 5 percent.

\section{Results}

\section{Changes in muscle oxygenation level caused by performing squat exercises}

Figure $2 \mathrm{~A}$ shows the changes in muscle oxygenation after performing a single squat at $50 \%$ of MLW. Muscle oxygenation in both groups decreased with the start of the squat movement, and after reaching the lowest peak value, it tended to gradually recover to the original level at resting (Figure $2 \mathrm{~A}$ ). The lowest peak value during the decrease in muscle oxygenation was $84.3 \pm 5.6 \%$ for the Control group and $68.1 \pm 8.1 \%$ for the Training group, respectively (Figure $2 \mathrm{~A}$ ). Similar results were obtained after performing a single squat at $80 \%$ of the participants' MLW (Figure 2B). The muscle oxygenation level observed immediately after exercise was $89.8 \pm 5.7 \%$ for the Control group and $84.0 \pm 3.1 \%$ for the Training group, respectively (Figure $2 \mathrm{~B}$ ). The lowest peak value during the decrease in muscle oxygenation was $65.1 \pm 9.8 \%$ for the Control group and $58.3 \pm 9.2 \%$ for the Training group, respectively (Figure $2 \mathrm{~B}$ ). On the other hand, during the multiple squats, a muscle oxygenation level
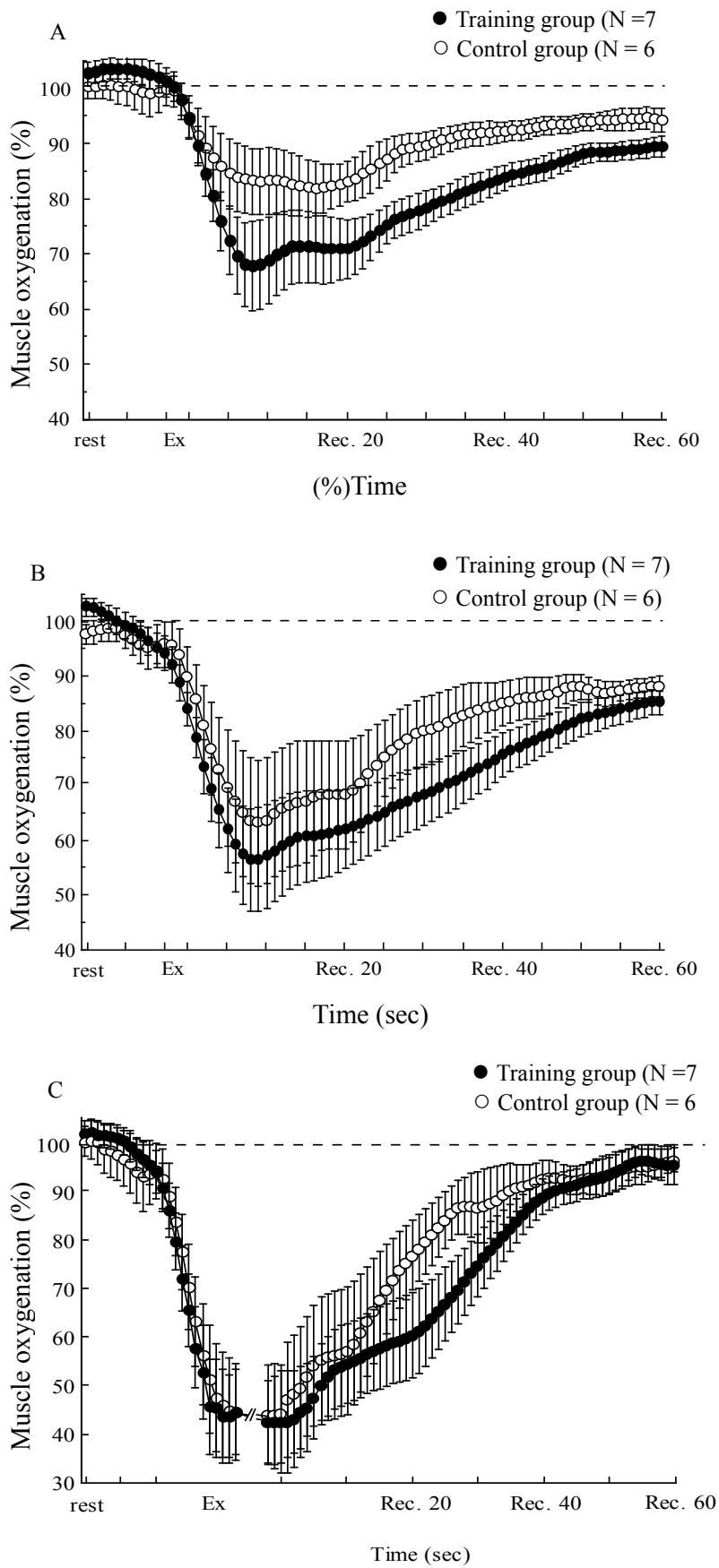

Figure 2: Changes in muscle oxygenation level caused by performing squat exercises

Changes in muscle oxygenation during one trial squat exercise with $50 \%$ (A) and $80 \%(B)$ of MLW and a recovery period in the Training (close circle) and Control (open circle) groups is shown. Values are means \pm SE. Rec=Recovery. $\mathrm{C}$ : Changes in muscle oxygenation during squat exercises to exhaustion with $80 \%$ of MLW and recovery period in the Training (close circle) and Control (open circle) groups. Values are means \pm SE. Rec $=$ Recovery.

at $80 \%$ of the participants' MLW decreased in a linear fashion followed by a stable condition in both groups (Figure 2C). After performing the movement, recovery to the original level at resting was time-dependent (Figure 2C). However, although the Control group's recovery time of muscle oxygenation was quick (especially between 10 and 25 seconds), the Training group showed a more gradual recovery rate during the same 
Citation: Aita F, Haga S, Sato S, Sakurai T, Esaki K, et al. (2013) Effects of Resistance Exercise on Intramuscular Oxygenation and Muscle Fiber Composition. J Sports Med Doping Stud 3: 133. doi:10.4172/2161-0673.1000133

Page 4 of 7

time period, which indicated a delay in recovery (Figure 2C).

\section{Changes in the rate of decrease in muscle oxygenation level caused by performing a single squat}

With the results shown in Figures $2 \mathrm{~A}$ and $2 \mathrm{~B}$, the rate of decrease in muscle oxygenation after performing a single squat at 50 and $80 \%$ of the participants' MLW was examined (Figure 3). At both loads, the rate of decrease in muscle oxygenation after performing a squat was significantly higher $(\mathrm{p}<0.05)$ in the Training group than in the Control group. The rate of decrease in muscle oxygenation in the Training group was significantly higher $(\mathrm{p}<0.01)$ at $80 \%$ of MLW than at $50 \%$ of MLW.

\section{Changes in recovery time of muscle oxygenation after multiple squats}

After performing multiple squats at $80 \%$ of the participants' MLW until exhaustion, recovery of muscle oxygenation was slower in the

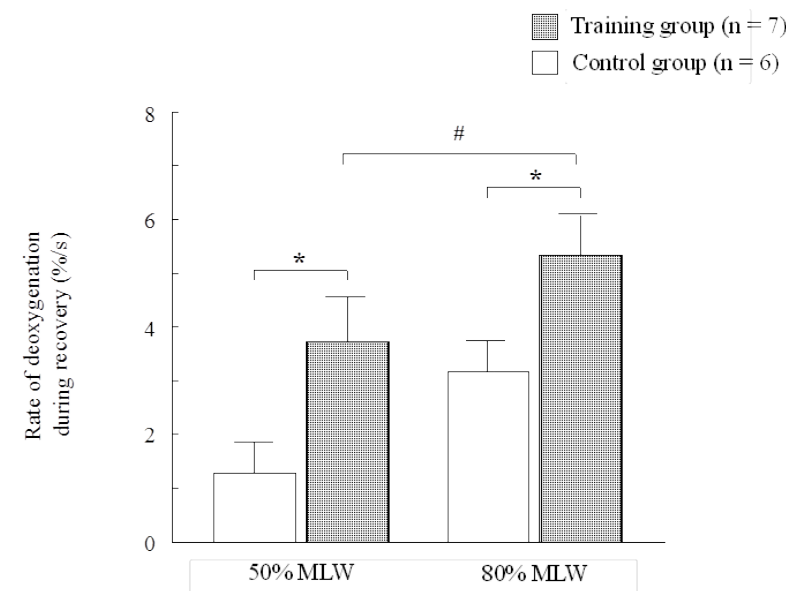

Figure 3: Changes in the rate of deoxygenation obtained during recovery Rate of deoxygenation obtained during recovery (\%/s) after both one trial squat exercise $(50 \%$ and $80 \%$ of MLW) was calculated. Values are means \pm SE. " $p<0.05$ vs. Control group; \# $p<0.01$ vs. $50 \%$ of MLW in Training group.

Training group $(\mathbf{n}=7)$

Control group $(\mathbf{n}=6)$

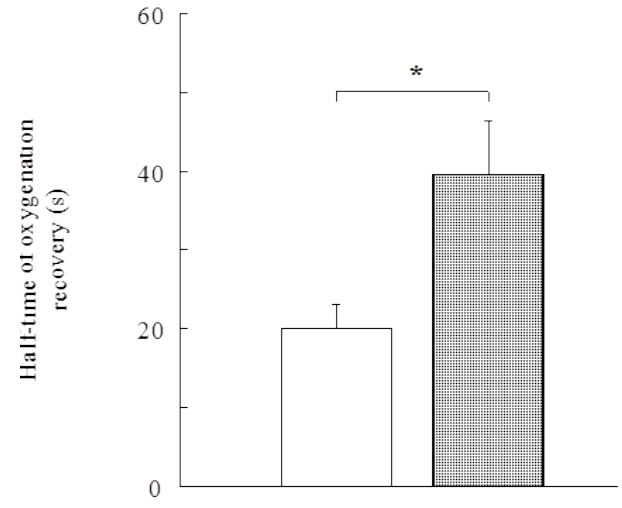

Figure 4: Changes in half-time of oxygenation recovery after squat exercises

Half-time of oxygenation recovery $\left(T_{1 / 2}\right)$ after squat exercises to exhaustion with $80 \%$ of MLW was calculated. Values are means \pm SE. ${ }^{*} p<0.05$ vs. Control group.
(A) Fiber type composition (\%)

\begin{tabular}{|l|c|c|c|}
\hline Fiber type & $\begin{array}{c}\text { Control group } \\
\mathrm{N}=6\end{array}$ & $\begin{array}{c}\text { Training group } \\
\mathrm{N}=7\end{array}$ & P-value \\
\hline Type I & $41.7 \pm 3.9$ & $47.8 \pm 6.9$ & NS \\
\hline Type Ila & $33.9 \pm 3.8$ & $41.1 \pm 6.4$ & NS \\
\hline Type Ilx & $23.6 \pm 3.6$ & $10.0 \pm 4.5$ & NS \\
\hline Type Ilc & $0.8 \pm 0.4$ & $1.0 \pm 0.7$ & NS \\
\hline
\end{tabular}

(B) Fiber type area distributuion (\%)

\begin{tabular}{|c|c|c|c|}
\hline Fiber type & $\begin{array}{c}\text { Control group } \\
N=6\end{array}$ & $\begin{array}{l}\text { Training group } \\
\qquad N=7\end{array}$ & P-value \\
\hline Type I & $41.9 \pm 4.4$ & $44.8 \pm 6.7$ & NS \\
\hline Type Ila & $36.7 \pm 4.1$ & $45.7 \pm 7.0$ & NS \\
\hline Type IIx & $20.8 \pm 2.9$ & $8.7 \pm 3.9$ & 0.05 \\
\hline Type Ilc & $0.7 \pm 0.4$ & $0.8 \pm 0.6$ & NS \\
\hline \multicolumn{4}{|c|}{ (C) Fiber type cross-sectional area $\left(\mu \mathrm{m}^{2}\right)$} \\
\hline Fiber type & $\begin{array}{l}\text { Control group } \\
\qquad N=6\end{array}$ & $\begin{array}{l}\text { Training group } \\
\qquad N=7\end{array}$ & P-value \\
\hline Type I & $4708 \pm 376$ & $5672 \pm 287$ & NS \\
\hline Type Ila & $5099 \pm 459$ & $6880 \pm 481$ & 0.05 \\
\hline Type IIx & $4207 \pm 447$ & $3004 \pm 1087$ & 0.05 \\
\hline Type Ilc & $2034 \pm 911$ & $1447 \pm 939$ & NS \\
\hline Mean & $4012 \pm 548$ & $4251 \pm 697$ & NS \\
\hline \multicolumn{4}{|c|}{ (D) Number of capillaries around fiber type } \\
\hline Fiber type & $\begin{array}{c}\text { Control group } \\
\qquad N=6\end{array}$ & $\begin{array}{l}\text { Training group } \\
\qquad N=7\end{array}$ & P-value \\
\hline Type I & $4.8 \pm 0.4$ & $5.6 \pm 0.3$ & NS \\
\hline Type Ila & $4.4 \pm 0.5$ & $5.4 \pm 0.2$ & NS \\
\hline Type IIx & $3.9 \pm 0.5$ & $2.3 \pm 0.8$ & NS \\
\hline Type Ilc & $1.2 \pm 0.6$ & $1.9 \pm 1.2$ & NS \\
\hline Mean & $3.6 \pm 0.5$ & $3.8 \pm 0.6$ & NS \\
\hline
\end{tabular}

Values are meals \pm SE

P-values denote level of significance between Training and Control groups

Table 2: Morphological characteristics of vastus lateralis muscle in Training and Control groups.

Mean area $\left(\mu \mathrm{m}^{2}\right)$

Control group Training group $P$-value $\mathrm{N}=6 \quad \mathrm{~N}=7$

\begin{tabular}{|l|l|l|l|}
\hline & $5.3 \pm 0.1$ & NS \\
\hline
\end{tabular}

\begin{tabular}{|l|l|l|l|l|}
\hline Number of capillaries per muscle fiber & $2.3 \pm 0.2$ & $2.7 \pm 0.1$ & 0.05
\end{tabular}

Number of capillaries per unit area $\quad 451.7 \pm 32.2415 .6 \pm 21.3 \quad$ NS

\begin{tabular}{l|l|l|l} 
Diffusion index $\left(\mu \mathrm{m}^{2}\right)$ & $2108 \pm 1462289 \pm 119$ & NS
\end{tabular}

Values are meals $\pm \mathrm{SE}$

P-values denote level of significance between Training and Control groups

Table 3: Mean Cross Sectional area of the vastus lateralis muscle in both groups.

Training group than in the Control group (Figure 2C). The time it took for the muscle oxygenation level to recover to $50 \%$ of its original value at rest $\left(\mathrm{T}_{1 / 2}\right)$ was examined. As a result, the delay in recovery was significantly longer $(\mathrm{p}<0.05)$ in the Training group than in the Control group (Figure 4).

\section{Muscle fiber composition}

As shown in Table 2A, the proportion of Type I and IIa fibers was not significantly different between both groups. The area distribution of Type IIx fiber was significantly lower $(\mathrm{p}<0.05)$ in the Training group than in the Control group (Table 2B). The cross-sectional area of Type IIa fiber was significantly higher $(\mathrm{p}<0.05)$ in the Training group than in the Control group, but that of Type IIx fiber was significantly lower $(\mathrm{p}<0.05)$ in the Training group than in the Control group (Table $2 \mathrm{C}$ ). The number of capillary vessels around each muscle fiber was not significantly different between the groups. 


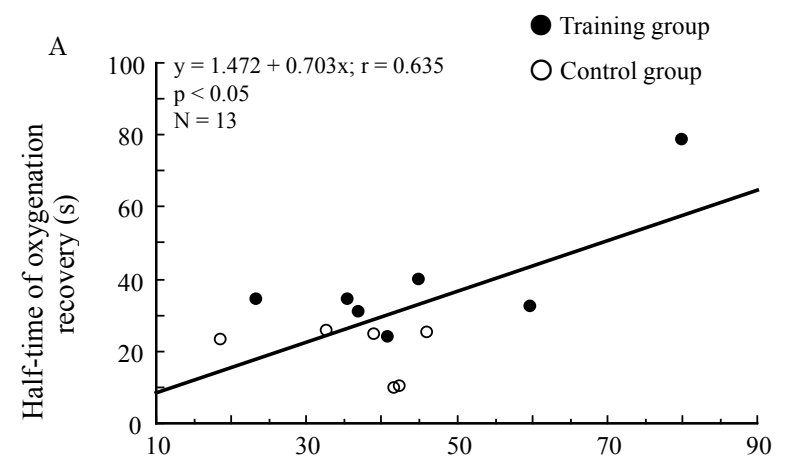

\% Type IIa

B

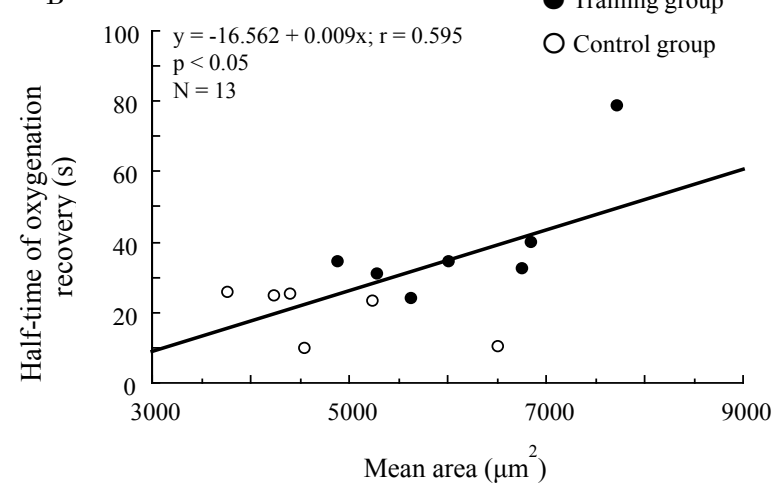

Figure 5: The relationship of the ratio of Type lla fibers and mean crosssectional area of muscle fiber with half-time of oxygenation recovery

A: Relationship between the \% Type Ila fibers and half-time of oxygenation recovery $\left(T_{1 / 2}\right)$ after squat exercises to exhaustion with $80 \%$ of MLW in Control and Training groups. Close and open circles indicate Training $(\mathrm{N}=7)$ and Control $(\mathrm{N}=6)$ groups, respectively. B: Relationship between the mean area of muscle fiber $\left(\mu \mathrm{m}^{2}\right)$ and half-time of oxygenation recovery $\left(T_{1 / 2}\right)$ after squat exercises to exhaustion with $80 \%$ of MLW in Control and Training groups. Close and open circles indicate Training $(\mathrm{N}=7)$ and Control $(\mathrm{N}=6)$ groups, respectively.

Table 3 shows the morphological characteristics of the vastus lateralis muscles. The mean value for the cross-sectional area of muscle fiber was significantly higher $(\mathrm{p}<0.05)$ in the Training group than in the Control group. Furthermore, the number of capillary vessels per muscle fiber was also higher $(\mathrm{p}<0.05)$ in the Training group than in the Control group. However, the number of capillary vessels per unit area was similar for both groups. Likewise, there was no difference in the diffusion index between the groups.

The relationship between the ratio of Type IIa fibers and $T_{1 / 2}$, and between the cross-sectional area of muscle fiber and $T_{1 / 2}$ after multiple squats

Finally, we examined the relationship of the ratio of Type IIa fibers and mean cross-sectional area of muscle fiber $\left(\mu \mathrm{m}^{2}\right)$ with $\mathrm{T}_{1 / 2}$. Significantly positive correlations were observed both between the ratio of Type IIa fibers and $\mathrm{T}_{1 / 2}$ (Figure $5 \mathrm{~A}$ ), and between the mean crosssectional area of muscle fiber and $\mathrm{T}_{1 / 2}$ (Figure $5 \mathrm{~B}$ ).

In the present study, a decrease in muscle oxygenation that depended on exercise intensity was observed after performing a single squat exercise for both the Training and the Control groups, even though the muscle fiber compositions were different between the two groups. However, this decrease was more remarkable in the Training group than in the Control group. Furthermore, the rate of decrease in muscle oxygenation was significantly higher in the Training group than in the Control group. In addition, in the Training group, the decreased muscle oxygenation level due to performing multiple squat exercises showed a delay in recovery at resting time, and as the ratio of the Type IIa muscle fiber increased, this phenomenon became more significant.

\section{Discussion}

The decrease in muscle oxygenation level in the Training group was significantly higher at $80 \%$ of the participants' MLW than at $50 \%$ of the participants' MLW. Also, even though it was not statistically significant, the Control group appeared to show a higher rate of decrease in muscle oxygenation at $80 \%$ of MLW than at $50 \%$ of MLW. The physiological reactions of the vastus lateralis muscle in the right thigh caused by performing a squat included a constriction of arterioles and blood capillaries due to an increase in intramuscular pressure associated with muscular contraction, microcirculatory dysfunction, and decreased peripheral muscle blood flow. These reactions appeared to become stronger as the exercise load increased.

The changes in the types of muscle fibers recruited were associated with the changes in the capacity of oxygen consumption by mitochondria, which brought about changes in muscle oxygenation. Oxygen consumption of mitochondria is thought to be governed by $\mathrm{ADP}, \mathrm{Pi}, \mathrm{NADH}$, and oxygen concentration and oxygen consumption by mitochondria increases according to an increase in the intensity of exercise $[27,28]$. In fact, Haga et al. [21] have reported that, when a high intensity exercise is performed, oxygen consumption by mitochondria increases and muscle oxygenation decreases from the beginning of the exercise. It can be assumed that the higher rates of decreases in muscle oxygenation observed at $80 \%$ of the participants' MLW in both groups were partly associated with the changes in the types of muscle fiber recruited due to the increase in exercise load. In both groups, activities employing Type I fiber might be significant at $50 \%$ of the participants' MLW. However, changes were seen in the rate of decrease in muscle oxygenation at $80 \%$ of MLW. It can be assumed that this occurred because activities employing Type IIa fibers, which have a relatively higher capacity of oxygen consumption than Type IIx fibers, were also prompted during the exercise performed at $80 \%$ of MLW.

On the other hand, the rate of decrease in muscle oxygenation after performing a single squat movement at both loads was significantly higher in the Training group than in the Control group. We may assume that the amount of oxygen consumption increased in the Training group because the cross-sectional area of skeletal muscle is larger in the Training group compared to the Control group, leading to more increased muscle oxygenation. $\mathrm{T}_{1 / 2}$ after performing multiple squats at $80 \%$ of the participants' MLW until exhaustion showed a significant delay in the Training group. The recovery time of muscle oxygenation level after exercise reflects the synthesis of phosphocreatine that decreased due to exercise and oxygen transport to mitochondria, as well as the speed of oxidative ATP resynthesis [22]. Therefore, this time factor is considered to be an index of the aerobic capacity of skeletal muscle [18].

To search for the causes underlying the differences in the recovery time of muscle oxygenation between the Control and Training groups, we further examined the muscle fiber composition in both groups. The results of this study suggest that with resistance training, increases in occupancy depend on the number and size of Type I and IIa fibers that result from the migration caused not only by an increase in the number and size of Type I and IIa fibers, but also by a decrease in the number and size of Type IIx fibers. It is well known that an increase in the amount 
of physical activity transitions into a slow-twitch type of muscle fiber, and, conversely, a decrease in the amount of physical activity transitions into a fast-twitch type of muscle [29]. Since subjects in the Training group of this study had habitually performed squat exercises, their Type I and IIa fibers were mainly stimulated and hypertrophied according to the "Principle of size" [6,30]. Furthermore, it is accepted that highintensity endurance training and sprint training promote the migration of the Type II fiber subtype (decrease in Type IIx fiber and increase in Type IIa fiber) which is the opposite effect of detraining [16,3133]. Actually, cast immobilization and bed rest is known to decrease the cross-sectional area of Type I fibers and conversely increase that of Type IIx fibers [34]. These reports demonstrate that the amount of physical activity that is defined by either intensity or endurance can be associated with the resultant transition to fiber type. Moreover, in the present study, because an increase in the cross-sectional area of Type IIa fiber was observed in the Training group, and there was significantly positive correlations between the ratio of Type IIa fibers and $\mathrm{T}_{1 / 2}$. One of the reasons for the delay in oxygenation recovery in the Training group is thought to be due to increased cross-sectional area of the Type IIa fiber.

The present study also showed that resistance training relatively, but not significantly, increased the number of capillary vessels around Type I and IIa fibers, and conversely decreased that around Type IIx fibers. The results from this study agreed with those from Schantz [26]. However, it is an accepted fact that intramuscular hypoxia induces the growth of capillary vessels and angiogenesis [35]. In particular, endurance training is known to increase the capillary plexus [24]. Additionally, since the endurance training-induced formation of the capillary plexus is recognized as being much higher under a hypoxic environment than under a low ground environment exercise-induced hypoxic stimulus to muscle tissue should contribute to the growth of the capillary plexus [36].

In summary, in the present study, we compared the differences in muscle fiber compositions and their relationship with muscle oxygenation levels between powerlifters, who regularly engaged in muscular resistance training, and ordinary healthy people. As a result, the present study demonstrates that habitual muscular resistance training increases the cross-sectional area of Type IIa fibers, but exhibits a delay in recovery of decreased muscle oxygenation level due to performing squat exercises. These results suggest that habitual muscular resistance training increases the amount of oxygen consumption because the cross-sectional area of Type IIa fiber is larger, compared to that found in ordinary healthy people.

\section{Acknowledgements}

This work was supported, in whole or in part, by the Grants-in-Aid for Specific Project Research from the Ministry of Education, Culture, Sports, Science and Technology of Japan, and the grant of THE DESCENTE AND ISHIMOTO MEMORIAL FOUNDATION FOR THE PROMOTION OF SPORTS SCIENCE.

\section{References}

1. MacDougall JD, Sale DG, Alway SE, Sutton JR (1984) Muscle fiber number in biceps brachii in bodybuilders and control subjects. J Appl Physiol Respir Environ Exerc Physiol 57: 1399-1403.

2. Moritani T, Sherman WM, Shibata M, Matsumoto T, Shinohara M (1992) Oxygen availability and motor unit activity in humans. Eur J Appl Physiol Occup Physiol 64: 552-556.

3. Sundberg CJ (1994) Exercise and training during graded leg ischaemia in healthy man with special reference to effects on skeletal muscle. Acta Physiol Scand Suppl 615: 1-50

4. Takarada Y, Nakamura Y, Aruga S, Onda T, Miyazaki S, et al. (2000) Rapid increase in plasma growth hormone after low-intensity resistance exercise with vascular occlusion. J Appl Physiol (1985) 88: 61-65.
5. Takarada $Y$, Sato $Y$, Ishii N (2002) Effects of resistance exercise combined with vascular occlusion on muscle function in athletes. Eur J Appl Physiol 86: 308314

6. Beachle TR, Earle RW (2000) Essentials of strength training and conditioning Human Kinetics, Champaign, Illinois.

7. Trappe SW, Costill DL, Fink WJ, Pearson DR (1995) Skeletal muscle characteristics among distance runners: a 20 -yr follow-up study. J Appl Physiol (1985) 78: 823-829.

8. Takarada Y, Ishii N (1998) Resistance training under hypoxia. J Health Phys Edu Recreation 48: 36-42.

9. Ozyener $F$ (2002) Evaluation of intra-muscular oxygenation during exercise in humans. Journal of Sports Science and Medicine 1: 15-19.

10. Quaresima V, Lepanto R, Ferrari M (2003) The use of near infrared spectroscopy in sports medicine. J Sports Med Phys Fitness 43: 1-13.

11. Hamaoka T, McCully K, Chance B, Iwane H (1994) Noninvasive measures of muscle metabolism. In: Exercise and Oxygen Toxicity. Sen C, Packer L, and Hanninen O, Elsevier Science, (Eds.), Amsterdam, 481-510.

12. Hoffman JR, Im J, Rundell KW, Kang J, Nioka S, et al. (2003) Effect of muscle oxygenation during resistance exercise on anabolic hormone response. Med Sci Sports Exerc 35: 1929-1934.

13. Hoffman JR, Im J, Kang J, Maresh CM, Kraemer WJ, et al. (2007) Comparison of low- and high-intensity resistance exercise on lipid peroxidation: role of muscle oxygenation. J Strength Cond Res 21: 118-122.

14. Rittweger J, Moss AD, Colier W, Stewart C, Degens H (2010) Muscle tissue oxygenation and VEGF in VO-matched vibration and squatting exercise. Clin Physiol Funct Imaging 30: 269-278

15. Spiering BA, Kraemer WJ, Hatfield DL, Vingren JL, Fragala MS, et al. (2008) Effects of L-carnitine L-tartrate supplementation on muscle oxygenation responses to resistance exercise. J Strength Cond Res 22: 1130-1135.

16. Wong del P, Tan EC, Chaouachi A, Carling C, Castagna C, et al. (2010) Using squat testing to predict training loads for lower-body exercises in elite karate athletes. J Strength Cond Res 24: 3075-3080.

17. Baechle TR, Earle RW (2008) Essentials of strength training and conditioning (3rdedn). Human Kinetics, Champaign, IL, 641.

18. Hamaoka T, Albani C, Chance B, Iwane H (1992) A new method for the evaluation of muscle aerobic capacity in relation to physical activity measured by near infrared spectroscopy. Med Sport Sci 37: 421-429.

19. Bae SY, Hamaoka T, Katsumura T, Shiga T, Ohno H, et al. (2000) Comparison of muscle oxygen consumption measured by near infrared continuous wave spectroscopy during supramaximal and intermittent pedalling exercise. Int $J$ Sports Med 21: 168-174.

20. Chance B (1992) Noninvasive approaches to oxygen delivery and cell bioenergetics in functioning muscle. Clin J Sport Med 2: 132-138.

21. Haga S, Bae SY, Hamaoka T, Katsumura T, Toshinai K, et al. (1998) Oxidative metabolism in skeletal muscle measured during supramaximal exercise in sprinter and active control groups by near infrared continuous wave spectroscopy. Adv Exerc Sports Physiol 4: 57-64.

22. Chance B, Dait MT, Zhang C, Hamaoka T, Hagerman F (1992) Recovery from exercise-induced desaturation in the quadriceps muscles of elite competitive rowers. Am J Physiol 262: C766-775.

23. Hamaoka T, Mizuno M, Katsumuea T, Osada T, Shimomitsu T, et al. (1998) Correlation between indicators determined by near infrared spectroscopy and muscle fiber types in humans. Jpn J Appl Physiol 28: 339-344.

24. Saltin B, Gollnick PD (1993) Skeletal muscle adaptability. In Handbook of Physiology, Skeletal muscle, Significance for metabolism and performance. Am Physiol Soc 555-631.

25. Brooke $\mathrm{MH}$, Kaiser KK (1970) Three "myosin adenosine triphosphatase" systems: the nature of their $\mathrm{pH}$ lability and sulfhydryl dependence. J Histochem Cytochem 18: 670-672.

26. Schantz P (1983) Capillary supply in heavy-resistance trained non-postura human skeletal muscle. Acta Physiol Scand 117: 153-155. 
Citation: Aita F, Haga S, Sato S, Sakurai T, Esaki K, et al. (2013) Effects of Resistance Exercise on Intramuscular Oxygenation and Muscle Fiber Composition. J Sports Med Doping Stud 3: 133. doi:10.4172/2161-0673.1000133

Page 7 of 7

27. Chance B, Leigh JS Jr, Kent J, McCully K, Nioka S, et al. (1986) Multiple controls of oxidative metabolism in living tissues as studied by phosphorus magnetic resonance. Proc Natl Acad Sci U S A 83: 9458-9462.

28. Mahler M (1985) First-order kinetics of muscle oxygen consumption, and an equivalent proportionality between $\mathrm{QO} 2$ and phosphorylcreatine level. Implications for the control of respiration. J Gen Physiol 86: 135-165.

29. Wada M, Katsuta S (1989) Muscle fiber performance. Jpn J Sports Sci 8: 62-68.

30. Sale DG (1987) Influence of exercise and training on motor unit activation. Exerc Sport Sci Rev 15: 95-151.

31. Andersen P, Henriksson $\mathrm{J}$ (1977) Capillary supply of the quadriceps femoris muscle of man: adaptive response to exercise. J Physiol 270: 677-690.
32. Allemeier CA, Fry AC, Johnson P, Hikida RS, Hagerman FC, et al. (1994) Effects of sprint cycle training on human skeletal muscle. J Appl Physiol (1985) 77: 2385-2390.

33. Glass DJ (2005) Skeletal muscle hypertrophy and atrophy signaling pathways Int J Biochem Cell Biol 37: 1974-1984.

34. Häggmark T, Eriksson $E$, Jansson $E$ (1986) Muscle fiber type changes in human skeletal muscle after injuries and immobilization. Orthopedics 9: 181-185.

35. Hudlicka O, Brown M, Egginton S (1992) Angiogenesis in skeletal and cardiac muscle. Physiol Rev 72: 369-417.

36. MacDougall JD (1994) Morphological changes in human skeletal muscle following strength training and immobilization. Champaign, IL, 269-288. 